\title{
La depresión tras el infarto agudo de miocardio
}

\author{
E. GARCÍA VICENTE, V. DEL VILLAR SORDO ${ }^{1}$, E. L. GARCÍA Y GARCÍA² \\ Unidad de Cuidados Intensivos. ${ }^{I}$ Servicio de Medicina Interna. Hospital Santa Bárbara. \\ Soria. ${ }^{2}$ Servicio de Rehabilitación y Medicina Física. Hospital Universitario Miguel \\ Servet. Zaragoza
}

POST-MYOCARDIAL INFARCTION DEPRESSION

\begin{abstract}
RESUMEN
La depresión mayor es un cuadro común entre pacientes convalecientes de un infarto agudo de miocardio (IAM). Además, síntomas depresivos clínicamente significativos se hallan presentes en otros pacientes cuya severidad sintomática o duración no reúne suficientes criterios establecidos para configurar el diagnóstico de depresión mayor. Durante la pasada década, la evidencia creciente sugiere que junto a su efecto sobre la calidad de vida del paciente, la depresión post-infarto también merece una atención especial ya que se ha descrito un incremento de morbilidad y mortalidad asociadas. Este artículo revisa los estudios que han estudiado la depresión o los síntomas depresivos en pacientes que han sufrido un infarto agudo de miocardio y se centra en la prevalencia, significación clínica, tratamiento, y métodos de evaluación de este cuadro. Un amplio número de estudios han evaluado varios aspectos de la depresión post-infarto incluyendo la prevalencia, su asociación con mortalidad, efectos adversos mayores y tratamiento.
\end{abstract}

PALABRAS CLAVE: Depresión. Infarto agudo de miocardio. Depresión post-infarto.

\begin{abstract}
Major depression is a common finding among patients recovering from a myocardial infarction. Additionally, clinically significant depressive symptoms are present in other patients whose symptom severity or duration does not meet established criteria for a diagnosis of major depression. Over the last decade, increasing evidence suggests that in addition to its effect on patient's quality of life, post-MI depression also deserves attention because of a reported relation to increased morbidity and mortality. This evidence report reviews the studies that have studied depression or depressive symptoms in patients after an MI and focuses on the prevalence, clinical significance, treatment, and methods of evaluating this condition. A large number of studies have evaluated various aspects of post-MI depression including prevalence, its association with mortality, and major adverse events, and treatment.
\end{abstract}

KEY WORDS: Depression. Myocardial acute infarction. Post-myocardial infarction depression.

García Vicente E, del Villar Sordo V, García y García EL. La depresión tras el infarto agudo de miocardio. An Med Interna (Madrid) 2007; 24: 346-351.

\section{INTRODUCCIÓN}

La depresión es un factor de riesgo de morbi-mortalidad cardíaca en pacientes con cardiopatía isquémica, especialmente tras un infarto agudo de miocardio (1). Hasta tal punto se ha asociado a la cardiopatía isquémica que se ha relacionado con un incremento 4 veces mayor en el riesgo de mortalidad durante los primeros 6 meses después de un infarto agudo de miocardio, y su significado pronóstico es comparable a poseer historia de disfunción ventricular izquierda en el seno de un infarto (2) y, según algunos estudios al grado de Killip, la fracción de eyección o el pico de creatin kinasa (3), si bien no todos los estudios prospectivos observacionales han encontrado una asociación entre depresión posterior a un infarto agudo de miocardio y mortalidad (4). Ante dicho debate, la hipótesis más razonable es que el grado de severidad de la enfermedad puede actuar como factor de confusión, dependiendo la exactitud de las percepciones del paciente de la severidad de su patología y de la condición en que se encuentre.

Ziegelstein ha señalado que aproximadamente uno de cada seis pacientes experimenta depresión mayor luego de un IAM, y 1 de cada 3 presentan síntomas depresivos durante la hospitalización (5). Un estudio de seguimiento de Lesperance et al. determinó que un $31,5 \%$ de pacientes infartados experimentó depresión durante la hospitalización y el primer año posterior al alta. Del total de pacientes con depresión, 50\% la presentó durante hospitalización, 42,9\% entre el alta y 6 meses, y 7,1\% entre 6 y 12 meses después del IAM. Por otra parte, aquellos pacientes que tenían el antecedente de depresión, tuvieron un riesgo mayor de sufrir un nuevo episodio de depresión post IAM, tanto en el hospital como después del alta (6). 
De todos modos, la depresión se encuentra con frecuencia infradiagnosticada e infratratada en pacientes con enfermedad cardiovascular. Se estima que sólo el $25 \%$ o menos de los pacientes cardíacos con depresión mayor están diagnosticados de esta última, y que sólo cerca de la mitad reciben tratamiento antidepresivo (7), gracias todo ello a los siguientes factores: a) algunos síntomas, como el insomnio y la fatiga, son comunes a la depresión y a la enfermedad coronaria; b) los médicos y los pacientes pueden erróneamente pensar que la depresión es una reacción normal a la enfermedad cardiovascular; c) los médicos pueden ser reacios a preguntar a sus pacientes acerca de sus posibles síntomas depresivos; d) los pacientes pueden ser remisos a comentar sus síntomas depresivos en el contexto de su enfermedad cardíaca; y e) los médicos pueden preferir no administrar tratamientos antidepresivos por miedo a sus posibles efectos adversos.

Entre los pacientes con infarto agudo de miocardio (IAM), los síntomas depresivos son factores predictivos independientes de mortalidad cardíaca, hospitalización, empeoramiento del estado de salud del enfermo y mayores costes sanitarios incluso en ausencia de diagnóstico clínico de depresión (8), demostrando Lauzon et al. que existía una diferencia estadísticamente significativa en la mortalidad a un año de ocurrido el IAM, entre aquellos pacientes que presentaban depresión al momento de la hospitalización y aquellos sin depresión (16 vs. $8 \%$ respectivamente) (9). No obstante, recientes estudios han desafiado a los mencionados. Mayou et al encontraron que los síntomas de depresión no predecían mortalidad pasado un año de un IAM en una muestra de 347 pacientes (10), aunque este y otros estudios se vieron limitados debido a que la mayor parte de ellos fueron realizados en centros en los cuales la generalización de los resultados es cuestionable.

Aún no se han identificado con precisión los factores por los que la depresión aumenta el riesgo de mortalidad en pacientes post IAM. Sin embargo, existen varias hipótesis entre las que se señala: a) aumento de la activación y agregación plaquetaria; b) aumento del tono adrenérgico que predispone a arritmias ventriculares; c) activación del sistema inmune, implicado tanto en el desarrollo de la placa ateroesclerótica como en el aumento de la mortalidad de pacientes post IAM; d) menor adherencia al tratamiento farmacológico y adopción de estilos de vida saludable; y e) menor soporte social (11), todo ello con el agravante de la posible coexistencia con algún trastorno psiquiátrico que puede complicar el diagnóstico, la evolución y el tratamiento de cualquier patología médica, junto con un aumento en los porcentajes y costos de atención (12). Para ello es de gran importancia el estudio de la prevalencia de depresión entre los pacientes hospitalizados, campo que se encarga de investigar la psiquiatría de enlace.

En un trabajo en el que se incluyó a 3.608 pacientes atendidos consecutivamente por las unidades de psiquiatría de enlace de cinco hospitales generales de la red pública española (Clínico de Barcelona, Clínico de Zaragoza, General de Alicante, Ramon y Cajal de Madrid y Princesa de Madrid) se estimó que entre el 30 y el $40 \%$ de los pacientes médicos hospitalizados presentaban comorbilidad psiquiátrica, siendo los diagnósticos psiquiátricos más frecuentes el trastorno depresivo primario (depresión mayor y distimia) o secundario al padecimiento de la enfermedad, y el trastorno mental orgánico (delirium y demencia). Como mínimo, el 10-14\% de los pacientes ingresados por una enfermedad médica presentaban síntomas de depresión (a diferencia de la población general, que presenta una prevalencia del 4-7\%) y el delirium aparece en el $10-15 \%$ de los pacientes hospitalizados por urgencias médicas o quirúrgicas (13).

Dentro de los pacientes ingresados por IAM, la depresión es un síntoma común, informándose que aproximadamente el $65 \%$ de los pacientes con infarto agudo de miocardio refieren síntoma depresivos, y entre el 15 y el $22 \%$ de ellos presentan un cuadro de depresión mayor (14). De cualquier modo, además de por su valor pronóstico, la depresión es un enfermedad importante de forma independiente, y una identificación precoz y tratamiento de la depresión en el período post-IAM está fuertemente indicado para todo tipo de pacientes, independientemente de su edad y sexo.

También se han descrito unos niveles más bajos de mejoría clínica en pacientes con cuadro depresivo y que han sufrido una intervención de by-pass coronario en un plazo de 6 meses, siendo el cuadro depresivo un factor de riesgo al menos tan decisivo como los parámetros tradicionales de función cardíaca (15).

En cuanto a distribución por sexos, se sabe que las mujeres padecen más depresión que los hombres, y amplios estudios epidemiológicos han mostrado que las mujeres mayores padecen menos depresión que las jóvenes. El sexo femenino presenta dos picos de aparición de depresión, uno en la pubertad y otro en la perimenopausia, hacia los 60 años, Y Vaccarino et al encontraron una supervivencia mayor en las mujeres de 60 o más años de edad que la de sus análogos masculinos.

Además, en la comunidad, las mujeres a edades cada vez más tempranas con diagnóstico de IAM tienen tasas de mortalidad mayores que los hombres sin poderse achacar este hecho a su historial médico, severidad clínica y procedimientos diagnóstico terapéuticos hospitalarios. Concretamente en el estudio de Vaccarino et al concerniente a el aumento de la tasa de mortalidad asociada al infarto de miocardio en mujeres jóvenes (por debajo de 50 años de edad), la tasa de mortalidad en mujeres era tres veces superior en mujeres que en hombres (20,9 vs. 13,6\%) (16). Tras un riguroso análisis de posibles causas clínicas y demográficas, además de la terapia cardiovascular administrada durante la hospitalización, no se encontró respuesta alguna para la mayor mortalidad presente en mujeres jóvenes, sugiriéndose factores genéticos, hormonales o inflamatorios, siendo la hipótesis más aceptada la vasoconstricción coronaria aumentada con un descenso de la utilización de oxígeno miocárdico debido a niveles aumentados de ADH plasmática y estrógenos (exógenos o endógenos) (16). Posiblemente los estrógenos son responsables de la existencia de niveles en plasma elevados de proteína $\mathrm{C}$ reactiva, circunstancia que se aprecia en muchos procesos inflamatorios y que parece ser que se asocia con una mayor incidencia de infarto de miocardio en mujeres (17), mientras que en lo referente a la hormona antidiurética, esta se asocia con un efecto vasoconstrictor que dificulta la oxigenación miocárdica (18).

Otro factor importante a tener en cuenta es la asociación de diabetes con depresión. La depresión es una patología común en diabéticos, particularmente mujeres, y al igual que en las no diabéticas, las mujeres presentan mayor aflicción que los hombres, y la prevalencia calculada de depresión clínicamente significativa en mujeres diabéticas es cercana al 28,2\% (19). Además, la depresión recurrente es común en la diabetes y el paciente promedio experimenta más de 4 episodios cada 5 años, incluso tras un tratamiento con éxito (20). 
Se ha objetivado que las tasas de depresión en mujeres hospitalizadas con IAM son de 1,5 a 2 veces más altas que en los hombres (21), pero se sabe muy poco sobre si las mujeres más jóvenes con IAM padecen más depresión que en otros grupos de edad. Sin embargo, y curiosamente, ese incremento en la prevalencia con respecto a los hombres no se manifiesta en una tasa sustancialmente mayor de mortalidad en la mujer.

Otro tema de imprescindible importancia es el de los instrumentos o métodos utilizados como cribaje de la depresión en el postinfarto. Se necesitan métodos exactos y fiables como screening en los pacientes que han sufrido un infarto agudo de miocardio. Estos métodos han de poseer una adecuada validez, fiabilidad y utilidad diagnóstica dentro de estas poblaciones de enfermos. Las características específicas de las herramientas de evaluación de grupos poblacionales son de especial relevancia en los supervivientes de un infarto agudo de miocardio debido a que los síntomas somáticos de depresión se confunden con frecuencia, y en ocasiones se superponen, con las secuelas físicas del infarto. Además, el momento en que se evalúa al paciente puede ser de vital importancia, ya que la propia hospitalización puede afectar y de hecho lo hace al sueño, el apetito, y otra serie de aspectos fundamentales de cara a evaluar a un paciente deprimido. Se deben tener presentes tres conceptos fundamentales: a) se logra una adecuada validez de un instrumento de medida si el instrumento es capaz de medir la depresión y diferenciarla de otras entidades similares aunque distintas; b) la fiabilidad se refiere al grado de consistencia de una medida; y c) la utilidad diagnóstica se refiere al grado en el cual una medida indentifica correctamente a los individuos que reúnen o no ciertos criterios diagnósticos determinados por un "gold standard" que para la depresión se podría considerar un correcto y estructurado proceso de entrevista. Validez, fiabilididad, y utilidad diagnóstica son conceptos multidimensionales que ganan fuerza con la convergencia de la evidencia. No pueden ser establecidos globalmente para un instrumento en particular, pero sí se definen en términos de grupos específicos poblacionales y el propósito para el cual se usan (p. ej. screening frente a búsqueda diagnóstica).

Numerosos instrumentos de medición se han usado para evaluar la depresión tras un infarto agudo de miocardio. Quizás los dos más utilizados sean el BDI y el HADS (22) tendiendo al parecer el primero a sobreestimar la prevalencia en los estudios. El BDI es una escala de autoevaluación compuesta por 21 items que miden actitudes características y síntomas de la depresión. El HADS es un autocuestionario de 14 items diseñado para detectar síntomas de ansiedad y depresión en una población no-psiquiátrica hospitalizada.

Por último, habría que preguntarse lo siguiente: ¿Difiere de alguna forma el tratamiento cardiovascular en aquellos pacientes con infarto agudo de miocardio y depresión frente a los que no la sufren? Se pueden considerar tratamientos cardiovasculares los siguientes: revascularización (angioplastia o by-pass), inhibidores de la enzima conversora de la angiotensina, betabloqueantes, estatinas, agentes antiplaquetarios, y otros tratamientos recomendados por la American Heart Association o la American College of Cardiology (23).

Hay que decir que la respuesta a esta pregunta permanece sin contestar de manera esclarecedora, habiéndose publicado escasos estudios referentes a ella hasta el momento. En el futuro, los trabajos que se publiquen deberán hacer especial hincapié en si las diferencias terapéuticas se deben a la pres- cripción del facultativo o más bien a la aceptación del paciente, así como si las medidas no farmacológicas (incluyendo dieta, ejercicio y rehabilitación cardíaca) recomendadas a los postinfartados difieren entre pacientes con y sin depresión, junto con la adherencia al tratamiento y las medidas para mejorarla.

\section{TRATAMIENTO PSICOFARMACOLÓGICO EN DEPRESIÓN- POSTINFARTO}

\section{CONSIDERACIONES TERAPÉUTICAS GENERALES}

A modo de resumen la terapia cognitivo-conductual se debería considerar ante los siguientes supuestos:

-Depresión no severa.

-Depresión no crónica.

-Ausencia de componente psicótico.

- Respuesta positiva previa a la terapia cognitivo-conductual.

-Disponibilidad de dicha terapia.

- Contraindicación para el tratamiento médico.

-Recuperación no alcanzada con medicación de forma exclusiva.

-Existencia de circunstancias psicosociales que complican el cuadro.

El tratamiento antidepresivo se ha de considerar en las circunstancias siguientes:

-Depresión severa.

-Depresión crónica o recurrente.

- Presencia de rasgos psicóticos.

- Respuesta previa positiva a la medicación.

-Existencia de historia familiar de depresión.

- Incapacidad del paciente para realizar el trabajo requerido en las sesiones de psicoterapia.

A la hora de escoger un antidepresivo debemos siempre valorar la seguridad, tolerabilidad, eficacia, precio y simplicidad (24), y debemos combinar el tratamiento farmacológico con la psicoterapia cuando los síntomas depresivos sean severos, crónicos, en los que fracase cada terapia aislada, y si existen significativos problemas psicosociales.

\section{TRATAMIENTO PSICOSOCIAL DE LA DEPRESIÓN POST-IAM}

El tratamiento psicosocial más efectivo para la depresión en pacientes que han sufrido un infarto agudo de miocardio es la terapia cognitivo-conductual. Este método asume que los pacientes son participantes activos en el tratamiento y se producen interacciones recíprocas entre las cinco áreas clave en la terapia como son -medio ambiente, pensamientos, emociones, comportamiento y factores fisiológicos-. La terapia cognitivo-conductual hace especial énfasis en resolución de problemas prácticos a corto plazo, favoreciendo el desarrollo de estrategias para cambiar los aspectos estresantes de la vida del paciente que este puede controlar.

La ayuda del facultativo puede ser de gran importancia para orientar determinadas expectativas y creencias del paciente tras sufrir un infarto. Por ejemplo, es frecuente para el enfermo y su familia el creer que el realizar ejercicio físico tras el evento cardíaco puede ponerle en riesgo de sufrir una muerte súbita. Como resultado de ello, restringe su actividad física, lo cual enlentece la recuperación, mantiene la depresión y aumenta el riesgo de desarrollar otros problemas de salud. 
Identificar estas concepciones erróneas y proporcionar un adecuado soporte educacional puede conducir a una mejor adherencia al tratamiento por el paciente y a un mejor acercamiento a su comportamiento, estado de ánimo y salud.

En cuanto al comportamiento, el médico debería animar de forma razonable al paciente a participar en actividades que comporten la realización de ejercicio físico, aficiones y actividades sociales además de los programas específicos de rehabilitación cardíaca. Fisiológicamente, el uso regular de la respiración, relajación o imaginación visual puede ayudar al enfermo a reducir su ansiedad.

Por último, es crucial involucrar a los miembros de la familia en el proceso de rehabilitación de este tipo de pacientes. Con mucha frecuencia, ellos experimentan sentimientos de falta de ayuda, pérdida y depresión similares a los que presenta el paciente, y sus parejas pueden temer el retorno a la actividad diaria, incluyendo la actividad sexual, basándose en conceptos erróneos. Educar a los familiares de los pacientes y a sus parejas y proporcionarles adecuadas fuentes de información es crucial de cara lograr altas tasas de supervivencia y recuperación (25).

Un metaanálisis (26) estudió la cuestión de si la adición de medidas psicosociales mejoraba el curso evolutivo de un régimen rehabilitador estándar para pacientes con cardiopatía isquémica. Los autores utilizaron un concepto amplio de intervención psicosocial identificando 23 ensayos clínicos aleatorizados. Concluyeron que el añadir una serie de medidas terapéuticas psicosociales a regímenes estandarizados de rehabilitación cardíaca reducía la morbilidad y la mortalidad, el estrés psicológico, algunos factores de riesgo biológico.

\section{TRATAMIENTO FARMACOLÓGICO DE LA DEPRESIÓN-POST IAM}

La eficacia y los efectos adversos son consideraciones importantes cuando se elige un agente farmacológico para el tratamiento de una depresión que sigue a un infarto. De forma global, no existen grandes diferencias en cuanto a la eficacia de los diferentes antidepresivos de cara al tratamiento de los cuadros leves-moderados (27), pero sí existen diferencias significativas en cuanto a lo que son sus efectos secundarios, frente a los antidepresivos tricíclicos, con efectos hipotensores, proarrítmogénicos y sobre la conducción cardíaca (incluso en pacientes sin patología cardíaca). Las respuestas hipotensivas que ocurren en ocasiones con estos fármacos tienen un profundo efecto sobre la perfusión de un tejido miocárdico previamente comprometido. La hipotensión ortostática puede llegar a ser más pronunciada si cabe en pacientes que reciban tratamiento concomitante con betabloqueantes, inhibidores de la enzima conversora de la angiotensina, o en pacientes que toman diuréticos para tratamiento de hipertensión o insuficiencia cardíaca.

La influencia de los ATC sobre la conducción cardíaca es similar a la de la quinidina (antiarrítmico clase I), y de hecho son eficaces como antiarrítmicos clase I. Se llegó a pensar en un posible efecto benéfico de los ATC, pero el estudio CAST (28) demostró que la terapia con los antiarrítmicos clase I incrementaba las tasas de mortalidad después del IAM. Aproximadamente el $20 \%$ de de los pacientes con alteraciones de la conducción, como por ejemplo un bloqueo de rama derecha, presentaban complicaciones durante la terapia con ATC (29).
Por lo tanto, el uso de ATC puede considerarse contraindicado en el contexto de un síndrome coronario agudo y ha de usarse con mucha precaución, en el caso de que se utilice, cuando existe historia de infarto agudo de miocardio. El bupropion también se ha estudiado en cardiópatas. Se han observado cambios estadísticamente significativos en la presión arterial sin efectos objetivables sobre la conducción cardíaca o la contractilidad (30).

La importancia de las potenciales interacciones entre los antidepresivos y las medicaciones cardiovasculares debe considerarse al tratar la depresión en pacientes cardiópatas. Un cierto número de estos agentes actúan sobre los sustratos enzimáticos del sistema del citocromo $\mathrm{P} 450$, y estas interacciones pueden ser especialmente problemáticas en pacientes que son metabolizadores lentos (se estima que un $8 \%$ de la población de raza blanca). La Fluoxetina es un inhibidor de la isoforma 2D6, y puede disminuir la velocidad de metabolización de los betabloqueantes, produciendo bradicardia. Otras interacciones de relevancia clínica serían las que presentan los antidepresivos tricíclicos con aspirina y fluoxetina (aumentan los niveles de ATCs), warfarina (aumentan el tiempo de protrombina), antiarrítmicos como la quinidina y la flecainida (prolongan el intervalo QT), clonidina (atenúan el efecto antihipertensivo de la clonidina, exagerando la respuesta hipertensiva a la retirada de la clonidina) $(31,32)$.

Durante las pasadas dos décadas, los ISRSs han emergido como agentes de primera línea en el tratamiento de la depresión leve-moderada. Al contrario que sus predecesores los antidepresivos tricíclicos, los ISRSs han demostrado repetidamente su seguridad y tener unos efectos insignificantes sobre el sistema cardiovascular, incluso en casos de sobredosis. Los ISRSs no tienen virtualmente efectos sobre los canales de sodio o la conducción, y y no se han descrito, debido a este fenómeno fisiológico, ningún efecto proarritmógeno o antiarrítmico. En un estudio que enfrentaba los efectos cardiovasculares de paroxetina con la nortriptilina, la cual es uno de los antidepresivos tricíclicos más tolerables debido a su menor actividad anticolinérgica, la tasa de interrupción debida a un evento cardiovascular fue significativamente mayor en el grupo de la nortriptilina ( 7 de 40) que en el grupo de la paroxetina (1 de 41). Además, el estudio mostró un aumento significativo en la frecuencia cardíaca al pasar de decúbito a bipedestación en los sujetos que recibían nortriptilina (33).

De los nuevos fármacos, la Venlafaxina quizá sea el menos aconsejable en pacientes con enfermedad cardiovascular preexistente. Se han descrito aumentos estadísticamente significativos en el ritmo cardíaco y la presión arterial, propiciando que los fabricantes recomienden controles periódicos de presión arterial. Por el contrario, ha habido solamente artículos aislados que hacen referencia a la aparición de bradicardia secundaria a ISRSs, si bien la fiabilidad de dichos trabajos no era excesiva (34).

Los inhibidores selectivos de la recaptación de la serotonina han demostrado una eficacia clara en el tratamiento de la depresión (35) y presentan menor número de efectos secundarios a todos los niveles, pero especialmente a nivel cardiovascular, que otros antidepresivos (36).

La utilización de ISRSs puede conferir un efecto protector frente al infarto de miocardio. Este hecho podría atribuírse al efecto inhibidor que los ISRSs tienen sobre la activación plaquetaria mediada por serotonina, al efecto protector sobre el endotelio o bien posiblemente por la mejora de otros factores 
asociados con el riesgo incrementado de infarto en el contexto de un cuadro depresivo (37), como por ejemplo las modificaciones que se ejercen sobre el estilo de vida, y un mejor cumplimiento de la terapia farmacológica, dieta y ejercicio físico.

La serotonina es liberada normalmente por las plaquetas activadas, causando aumento de la agregación plaquetaria, lo cual contribuye a la patogénesis del infarto agudo de miocardio. Un polimorfismo genético del transportador de serotonina (el único mecanismo de captación de serotonina por parte de las plaquetas) genera una mayor captación de serotonina y se asocia con una mayor probabilidad de sufrir un evento coronario. Los antidepresivos, y particularmente los ISRSs (inhibidores selectivos de la recaptación de la serotonina), con una alta afinidad por el transportador de serotonina, atenúan la activación plaquetaria debido a una depleción de las reservas de serotonina (38) y han mostrado que disminuyen la actividad plaquetaria en pacientes coronarios. Se ha visto que un paciente tipo afecto de depresión mayor por lo general muestra alteraciones de diversos parámetros plaquetarios, incluyendo un aumento en el número de receptores 5HT2 serotonínicos en la superficie de las plaquetas, una elevación de la monoaminoxidasa plaquetaria, una elevación de la expresión de la glicoproteína GPIIb-IIIa y receptores $\mathrm{P}$ selectivos en membrana, una recaptación serotonínica plaquetaria menor, un incremento de la expresión de los receptores serotonínicos 5HT2 y una mayor movilización del calcio plaquetario como respuesta a la estimulación ejercida por la serotonina (39).

De cualquier forma, la evidencia no deja lugar a la duda. La serotonina es un elemento clave en la patogénesis de la enfermedad coronaria y el antagonismo serotonínico juega un papel primordial en la terapéutica cardiovascular, (40) una acumulación transcardíaca excesiva de serotonina parece jugar un papel trascendental en la conversión de angina estable crónica a síndrome coronario agudo (41), y se ha descrito que la serotonina, asociada a otras sustancias, tiene un papel mediador en la obstrucción coronaria intermitente causada por agregación plaquetaria y obstrucción dinámica. La activación plaquetaria conduce a una liberación local de serotonina, la cual estimula las terminaciones aferentes simpáticas. Este evento produce vasoconstricción u agregación plaquetaria recurrente con reducciones cíclicas en el flujo vascular (42).

La serotonina también es posible que actúe como factor de crecimiento que estimula la mitogénesis y la migración de células musculares arteriales lisas, factores ambos que tienen un efecto negativo tras las intervenciones percutáneas. Es más, en modelos animales de estenosis coronaria y lesión endotelial, los receptores antagonistas de la serotonina mos- traron una fuerte protección frente a la agregación plaquetaria repetitiva, incluso cuando los niveles de catecolaminas se encontraban marcadamente elevados (43). No obstante,a pesar del efecto antiplaquetario de los nuevos antidepresivos, la mayor parte de los cuales tienen una elevada afinidad por el transportador serotonínico, se ha visto también implicado en un riesgo aumentado de sangrado gastrointestinal (44). Un estudio reciente de van Walraven et al describe un incremento en el riesgo de hemorragia digestiva alta con el consumo de este tipo de fármacos (45).

No obstante, no todos los antidepresivos poseen la misma afinidad por el transportador serotonínico. Presentan una alta afinidad ( $\mathrm{Kd}<1 \mathrm{nmol})$ la Paroxetina, Clomipramina, Sertralina y Fluoxetina. Con una afinidad moderada (Kd 1-10 nmol) se encuentran el Citalopram, Imipramina, Fluvoxamina, Amitriptilina y Venlafaxina, y ya el grupo de antidepresivos con una baja afinidad ( $\mathrm{Kd}>10 \mathrm{nmol})$ serotonínca son la Desipramina, Nortriptilina, Protriptilina, Amoxapina, Doxepina, Trimipramina, Trazodona, Nefazodona, Maprotilina, Bupropion y Mirtazapina.

Posiblemente el estudio pionero en cuanto a valorar la seguridad o eficacia de los ISRSs en el post IAM o post angina inestable ha sido el reciente SADHART (Sertraline Antidepressant Heart Attack Randomized Trial) elaborado con 364 pacientes deprimidos con síndrome coronario agudo (46). En dicho estudio no se evidenció daño alguno en los pacientes tratados con Sertralina, es más, se observó una tendencia clara a la redución en la morbi-mortalidad en los pacientes tratados con Sertralina, todo ello a pesar de recibir tratamiento concomitante con aspirina y/o clopidogrel. Los resultados de este estudio también se extienden a la enfermedad cerebrovascular.

A pesar de todo, a largo plazo algunos efectos deletéreos cardiovasculares son posibles, y así se ha descrito una elevación de los niveles de lipoproteínas de baja densidad (47), quizá el principal factor de riesgo cardiovascular convencional, y particularmente, la paroxetina puede inducir a un marcado aumento de peso, otro factor de riesgo cardiovascular convencional (48).

También hay que decir que la FDA ha advertido del peligro de producir un síndrome serotoninérgico al asociar ISRSs y triptanos. El síndrome serotoninérgico tiene una repercusión clínica amplia, destacando la taquicardia y la hipertensión arterial como manifestaciones autonómicas, alteraciones en el estado mental y trastornos neuromusculares. El reconocimiento precoz es muy importante, porque es potencialmente fatal. El manejo incluye la retirada de los agentes causales y medidas de soporte como estabilización hemodinámica, sedación, control térmico, hidratación y monitorización de las complicaciones.

\section{Bibliografía}

1. Kauffmann MW, Fitzgibbons JP, Sussman EJ, et al.Relation between myocardial infarction, depresión, hostility, and death. Am Heart $\mathrm{H}$ 1999; 138: 549-54.

2. Frasure-Smith N, Lesperance F, Talajic M. Depresion following myocardial infarction: impact on 6 month survival. JAMA 1993; 273: 1819-25.

3. Bush DE, Ziegelstein RC, Tayback M, Richter D, Stevens S, Zahalsky $\mathrm{H}$, Fauerbach JA Even minimal symptoms of depression increase mortality risk after acute myocardial infarction. Am J Cardiol 2002; 1: 89: 112-3.

4. Lane D, Carroll D, Lip GY. Anxiety, depression, and prognosis after myocardial infarction: is there a causal association? J Am Coll Cardiol 2003; 19: 42: 1808-10.

5. Ziegelstein RC. Depression in patients recovering from a myocardial infarction. JAMA 2001; 286: 1621-7.

6. Lesperance F, Frasure-Smith N, Juneau M, Theroux P. Major depression before and after myocardial infarction: Its nature and consequences. Psychosom Med 1996; 58: 99-110.

7. Musselman DL, Evans DL, Nemeroff CB. The relationship of depression to cardiovascular disease: Epidemiology, biology, and treatment. Arch Gen Psychiatry 1998; 55: 580-92.

8. van Melle JP, de Jorge P, Spikerman T, et al. Prognostic association of depresión following myocardial infarction with mortality and cardiovascular events: a meta-analysis. Psychosom Med 2004; 66: 814-822.

9. Lauzon C, Beck CA, Huynh T, Dion D, Racine N, Carignan S, et al. 
Depression and prognosis following hospital admission because of acute myocardial infarction. CMAJ 2003; 168: 547-52.

10. Mayou RA, Gill D, Thompson DR, Day A, Hicks N, Volmink J, et al. Depression and anxiety as predictors of outcome after myocardial infarction.Psychosom Med 2000; 62: 212-9.

11. Ziegelstein RC, Fauerbach JA, Stevens SS, Romanelli J, Richter DP, Bush DE. Patients with depression are less likely to follow recommendations to reduce cardiac risk during recovery from a myocardial infarction.Arch Intern Med 2000; 160: 1818-23.

12. Levenson JL, Hamer RM, Rossiter LF. Relation of psychopathology in general medical inpatients to use and cost of services. Am J Psychiatry 1990; 147: 1498-503.

13. Valdés M, de Pablo J, Campos R, Farré JM, Girón M, Institut Clinic de Psiquiatria, et al. El proyecto multinacional europeo y multicéntrico español de mejora de calidad asistencial en psiquiatría de enlace en el hospital general: el perfil clínico en España. Med Clin 2000; 115: 690-4.

14. Guck TP, Kavan MG, Elsasser GN, Barone EJ. Assessment and treatment of depression following myocardial infarction. Am Fam Physician 2001; 15: 64: 641-8. Review.

15. Mallik S, Krumholz HM, Lin ZQ, Kasl SV, Mattera JA, Roumains SA, Vaccarino V. Patients with depressive symptoms have lower health status benefits after coronary artery bypass surgery.Circulation 2005; 25 : 111: 271-7. Epub 2005 Jan 17.

16. Vaccarino V, Parsons L, Every NR, Barron HV, Krumholz HM. Sexbased differences in early mortality alter myocardial infarction. N Engl J Med 1999; 341: 217-25.

17. Ridker PM, Hennekens CH, Buring JE, Rifai N. C-reactive protein and other markers of inflammation in the prediction of cardiovascular disease in women. N Engl J Med 2000; 23: 342: 836-43.

18. Kozniewska E, Roberts TP, Vexler ZS, Oseka M, Kucharczyk J, Arieff AI. Hormonal dependence of the effects of metabolic encephalopathy on cerebral perfusion and oxygen utilization in the rat.Circ Res 1995; 76: 551-8.

19. Anderson RJ, Freedland KE, Clouse RE, Lustman PJ. The prevalence of comorbid depression in adults with diabetes: A meta-analysis.Diabetes Care 2001; 24 (6): 1069-78.

20. Lustman PJ, Griffith LS, Freedland KE, Clouse RE. The course of major depression in diabetes. Gen Hosp Psychiatry 1997; 19: 138-43.

21. Frasure-Smith N, Lesperance F, Juneau M, Talajic M, Bourassa MG. Gender, depresión and one-year prognosis after myocardial infarction. Psychosom Med 1999; 61: 26-37.

22. Jacqueline JMH Strik MD, Adriaan Honig MD, PhD. MRC. Psych, Richel Lousberg PhD, Johan Denollet PhD. Sensitivity and Specificity of Observer and Self-Report Questionnaires in Major and Minor Depression Following Myocardial Infarction. Psychosomatics 2001; 42: 423-8.

23. The Johns Hopkins University Evidence-based Practice Center, Baltimore MD. Post-Myocardial Infarction depresión. Evidence Report/Technology Assessment. Number 123

24. Preskorn S. Outpatient management of depresión. $2^{\text {nd }}$ ed. Caddo, Okla: Profesional Communications, 1999.

25. Guck TP, Kavan MG, Elsasser GN, Barone EJ. Assessment and treatment of depression following myocardial infarction. Am Fam Physician 2001; 15: 64: 573

26. Linden W, Stossel C, Maurice J. Psychosocial interventions for patients with coronary artery disease: a meta-analysis. Arch Intern Med 1996; 156: 745-52.

27. Simon GE, VonKorff M, Heiligenstein JH, Revicki DA, Grothaus L, Katon W, et al. Initial antidepressant choice in primary care. Effectiveness and cost of fluoxetine vs. tricyclic antidepressants. JAMA 1996; 26: 275 : $1897-902$
28. Preliminary report: Effect of encainide and flecainide on mortality in a randomized trial of arrhytmia supresión after myocardial infarction. The Cardiac Arrhitmia Supresión Trial Investigators. N Engl J Med 1989 231: 406-12.

29. Tricyclic antidepressants in depressed patients with cardiac conduction disease. Arch Gen Psychiatry 1987; 44: 273-5.

30. Roose SP, Dalack GW, Glassman AH, Woodring S, Walsh BT, Giardina EG. Cardiovascular effects of bupropion in depressed patients with heart disease. Am J Psychiatry 1991; 148: 512-6.

31. Walley T, Pirmohamed M, Proudlove C, Maxwell D. Interaction of metoprolol and fluoxetine. Lancet 1993; 10: 341: 967-8.

32. Guck TP, Kavan MG, Elsasser GN, Barone EJ. Assessment and treatment of depression following myocardial infarctionAm Fam Physician. 2001; 15: 64: 641-8. Review.

33. Roose SP, Laghrissi-Thode F, Kennedy JS, Nelson JC, Bigger JT Jr, Pollock BG, et al. Comparison of paroxetine and nortriptyline in depressed patients with ischemic heart disease. JAMA 1998; 28: 279: 287-91

34. Ellison JM, Milofsky JE, Ely E. Fluoxetine-induced bradycardia and syncope in two patients. J Clin Psychiatry 1991; 52: 139.

35. Song F, Freemantle N, Sheldon TA, et al. Selective Serotonin reuptake inhibitors: meta-analysis of efficacy and acceptability. Br Med J 1993 306: 683-7

36. Roose SP, Laghrissi-Thode F, Kennedy JS, et al. Comparison of paroxetine in depressed patients with heart disease. Am J Psichiatry 1998 155: 660-5.

37. Sauer WH, Berlin J, Kimmel S. Selective Serotonin Reuptake Inhibitors and Myocardial Infarction. Circulation 2001; 104: 1894-8.

38. Hergovich N, Aigner M, Eichler HG, Entlicher J, Drucker C, Jilma B Paroxetine decreases platelet serotonin storage and platelet function in human beings. Clin Pharmacol Ther 2000; 68: 435-42.

39. Rasmussen A, Hindberg I, Mellerup E. Does sertraline induced platele dysfunction protect stroke patients against cardiovascular comorbidity? Int.J Neuropsychopharmacol 2000; 3 (Supl. 1).

40. Pakala R, Willerson JT, Benedict CR. Effect of serotonin, thromboxane A2, and specific receptor antagonists on vascular smooth muscle cell proliferation. Circulation 1997; 7: 96: 2280-6.

41. Vikenes K, Farstad M, Nordrehaug JE. Serotonin is associated with coronary artery disease and cardiac events Circulation 1999; 3: 100 483-9.

42. Ashton JH, Benedict CR, Fitzgerald C, Raheja S, Taylor A, Campbell $\mathrm{WB}$, et al. Serotonin as a mediator of cyclic flow variations in stenosed canine coronary arteries. Circulation 1986; 73: 572-8).

43. Pakala R, Willerson JT, Benedict CR. Effect of serotonin, thromboxane A2, and specific receptor antagonists on vascular smooth muscle cell proliferation. Circulation 1997; 7: 96: 2280-6.

44. Meijer WEE, et al. Association of risk of abnormal bleeding with degree of serotonin reuptake inhibition by antidepressants. Arch Intern Med 2004; 164: 2367-70

45. van Walraven C, Mamdani MM, Wells PS, Williams JI. Inhibition of serotonin reuptake by antidepressants and upper gastrointestinal bleeding in elderly patients: Retrospective cohort study. BMJ 2001; 22: 323 $655-8$.

46. Glassman AH, O'Connor CM, Califf RM, et al. Sertraline treatment of major depression in patients with acute myocardial infarction or unstable angina. JAMA 2002; 288: 701-9.

47. Lara et al, Increased cholesterol levels during paroxetine administration in healthy men. J Clin Psychiatry 2003; 64: 1455-9.

48. Dannon PN, Iancu I, Cohen A, Lowengrub K, Grunhaus L, Kotler M. Three year naturalistic outcome study of panic disorder patients treated with paroxetine. BMC Psychiatry 2004; 11: 4: 16 